\title{
Anti-mycobacterial compounds derived from marine organisms: Mode of actions and potential in tuberculosis treatment
}

\author{
Apsara S. Babu, K. V. Bhaskara Rao* \\ Marine Biotechnology Laboratory, Department of Biomedical Sciences, School of Biosciences and Technology, Vellore Institute of Technology, \\ Vellore, Tamil Nadu, India
}

Received 18th January 2021 / Accepted 24th March 2021

\begin{abstract}
Mycobacteria are an extremely large group of bacteria that are normal inhabitants of soil and water. It has an exceptional propensity to adjust to varying environmental conditions which has led to the success of this pathogen. Mycobacterium tuberculosis can inhabit human tissues for years without replicating, thereby resuming growth and resulting in active tuberculosis with an increased latency period compared to any other deadly disease. The existing vaccines and drugs in the market to treat tuberculosis are ineffective and pose severe side effects. Hence, the development of a novel drug with high efficacy to kill these replicating and non-replicating bacilli appears to be an attractive solution to tackle this problem. The extraction of novel biologically active compounds from marine organisms has prospective biomedical applications for future drug discovery. Tuberculosis treatment, in specific, may benefit from the identification of novel and efficient marine-based compounds. A few anti-mycobacterial compounds belonging to the class alkaloids, terpenoids, peptides, and steroids have been isolated from marine organisms and are known to have potential activity against mycobacteria. This review focuses on the currently available anti-mycobacterial compounds and quorum sensing inhibitors of marine origin along with their mode of action and their significant potential in the treatment of mycobacteria associated strains.
\end{abstract}

Keywords: Actinomycetes, antibiotics, anti-mycobacterial compounds, marine drugs, marine microorganisms

\section{INTRODUCTION}

Tuberculosis (TB) also called the white plague, is a bacterial infection caused by Mycobacterium tuberculosis. It is one of the major causes of mortality worldwide. Mycobacteria are aerobic, non-motile, non-capsulated, and non-sporing scanty rods that occasionally show branching resembling fungal mycelium. They are also known as acid-fast bacilli (AFB) as they resist decolorization and are highly tolerant to stress due to the presence of their impermeable cell wall.
Mycobacterial cell wall differs from other bacteria owing to its biochemical and structural characteristics. The majority of the early research focused on the understanding of cell wall biosynthesis. Advanced molecular techniques like whole-genome sequencing have increased the insight for future drug discovery (Jankute et al., 2015). One of the major distinctive features of the mycobacterial cell wall is the variety of lipids and carbohydrates that act as a permeability barrier

*Author for correspondence: K. V. Bhaskara Rao, Department of Biomedical Sciences, School of Biosciences and Technology, Vellore Institute of Technology, Vellore, Tamil Nadu, India. Email kvbhaskararao@vit.ac.in 
against hydrophilic molecules as well as antibiotics. Effective antibiotics for tuberculosis are thus laborious to develop (Quan et al., 2019). The cell envelope of $M$. tuberculosis consists of three major components: long-chain mycolic acids, an extremely branched arabinogalactan polysaccharide, and a cross-linked polymer of peptidoglycan. The intact mycolylarabinogalactan-peptidoglycan (mAGP) complex is essential for cell viability and virulence. In addition to that, inert waxes and glycolipids which form the outer membrane intercalate the mycolate layer of the mAGP complex. The outermost sheaths of the cell envelop are composed of proteins and polysaccharides (Bhat et al., 2017; Jankute et al., 2015). Therefore antimycobacterial agents that can specifically inhibit the assembly of cell wall constituents can be an attractive target for the treatment of TB (Abrahams et al., 2018). The culturing of mycobacterial strains in the laboratory is painstaking as they are slow-growing and produce visible colonies only after 10-28 days. Other complications associated with the study of mycobacteria involve the high risk of handling pathogens, cell aggregation, and resistance of cells to lysis (Cook et al., 2009).

TB is mainly a pulmonary disease even though it affects bone and joints, the central nervous system, and other organs. It is caused by the direct inhalation of bacilli in the droplet nuclei of cough containing sputum. The inhaled bacilli are then arrested at the upper respiratory tract by the natural defense mechanisms of the human body and on reaching the lungs are ingested by the alveolar macrophages. The pathogen can then endure in the body for several years as latent tuberculosis. TB in the latent form remains asymptomatic and initiate long-term infections. People infected with HIV are at major risk to develop active TB. Several other factors like host immune response, genetic susceptibility, age, immunocompetence, stress, and nutrition influence the outcome of the infection (Smith, 2003).

TB was assumed to be an untreatable and lethal disease before the establishment of antimycobacterial drugs. In 1960, the development of front line antibiotics has brought TB under benchmark to some extent (Pauli et al., 2005). The present-day treatment for tuberculosis consists of Bacille Calmette-Guerin (BCG) in combination with antibiotics like rifampicin, isoniazid, ethambutol, and pyrazinamide has reduced the duration of treatment from 18 to 6 months. Although anti-tuberculosis drugs have reduced mortality to some extent, the emergence of multidrug resistant, extensively-drug resistant, extremely-drug resistant, and drug resistant TB has become a key barrier in the treatment of this deadly disease (Hou et al., 2019). The principal challenge in TB treatment is the emanation of persisters as they are drug resistant. The ability of bacteria to persist within the host for a longer time results in biofilm formation (Sharma et al., 2014). Biofilm forms when bacteria adhere to surfaces in aqueous environments and collectively excrete a slimy glue-like substance called extracellular polymeric substance (EPS) that helps in anchorage to all kinds of surfaces. This social behaviour of bacteria is mediated by a process known as quorum sensing where bacterial cells communicate with each other by releasing, sensing, and responding to small diffusible molecules called autoinducers (Sharma et al., 2014; Arai et al., 2013). A possible way to interrupt this bacterial communication is by the use of quorum sensing inhibitors which have been validated as a novel therapeutic agent in treating bacterial infections (Miao et al., 2017).

Mycobacterial biofilms are troublesome as they are resistant to most antibiotics and of the host's immune system. The various factors that have been hypothesized to trigger biofilm formation include lipids in the mycobacterial cell wall, oxidative stress, presence of free mycolic acids, and depletion of iron in the medium (Padhi et al., 2016). Given the current scenario, it is important to identify a novel antimycobacterial compound from marine microorganisms with (1) advanced pharmacokinetic properties, (2) fewer side effects, and (3) short-term duration of treatment.

Nowadays, the search for novel pharmacologically active compounds has been focused on natural products because they are an effective and less toxic alternative to synthetic drugs (Riera et al., 2020). Due to the abundance and diversity of marine microorganisms, they have slowly risen to become an important source of bioactive compounds for biomedical applications. The rich biological and genomic diversity of marine organisms arise from their 
survival under harsh marine environmental conditions such as poor light, high pressure, and cold temperatures (Nikapitiya, 2012). Due to this unique habitat, marine microorganisms can produce novel secondary metabolites with biological activities.

The marine environment contains a vast range of microorganisms that are less explored in contrast to the terrestrial environment. Among the main groups of microorganisms are bacteria, fungi, microalgae, and symbiotic microorganisms. To date, more than 20,000 bioactive metabolites produced by marine microorganisms have been reported, and almost 10,000 are derived from actinomycetes, mainly of Streptomyces species (Bibi et al., 2020; Brinkmann et al., 2017). Many of these bioactive compounds isolated from marine microorganisms are diverse in their functions exhibiting anti-bacterial, anti-fungal, anti-tumor, anti-viral, and other biological activities (Bibi et al., 2020). This review emphasizes on the mechanism of action of marine-derived bioactive compounds and the interruption of quorum sensing pathways to control the bacterial growth.

\section{Isolation, identification and extraction of anti-mycobacterial compounds from marine organisms}

Marine organisms producing secondary metabolites can be isolated from different locations of the coastal region. (Prakash et al., 2010). According to the "one strain many compounds concept", a change of growth conditions can result in the chemical diversity of the fungal bioactive compounds. Therefore, various fermentation conditions like shaking versus static cultures, variation in temperature, light, and aeration can be implemented to obtain diverse bioactive compounds. Fermentation media such as potato dextrose agar, Czapek dox agar, barely spelled solid substrate, liquid Wickerham's media, malt extract medium have been used for the successful scale-up of bioactive compounds from fungi. Identification of fungal isolates can be achieved by cultural and morphological characters using lactophenol cotton blue stain.

The actinomycetes strain can be isolated and grown on Actinomycetes isolation agar (AIA). The cultural characteristics of the strain can be studied by performing various biochemical tests, visual examination of coloration, and nature of growth on ISP (International Streptomycetes project) media (2-7). Morphological evaluation was examined using a scanning electron microscope (Abrahams et al., 2018). Following fermentation the cell free supernatant medium can be subjected to dryness in a lyophilizer at $-80^{\circ} \mathrm{C}$.

Solvent extraction and chromatographic techniques like column chromatography, thinlayer chromatography (TLC), Sephadex column chromatography, High-performance liquid chromatography (HPLC) can be used for the purification of the bioactive compound. The purified compound can be characterized by chemical, biochemical, and biological analysis. Molecular characterization like 16s rRNA ribotyping and phylogenetic analysis can be carried out to identify the novelty of the compound. The structure of the compound can be analyzed using Fourier-transform infrared spectroscopy (FTIR), high-resolution electrospray ionization mass spectrometry, 1D and 2D nuclear magnetic resonance (NMR), gas chromatography mass spectrometry (GC-MS) (Siddharth et al., 2019). Toxicity against mammalian cells can be determined by MTT assay which can reveal the therapeutic potential of the compound.

Computational methods have significantly reduced the period and cost involved in the discovery of current drugs. In-silico molecular docking analysis made it easier to identify the drug target by protein-ligand docking analysis. In protein-ligand docking analysis, the lead compound can be analyzed in-silico for its ability to inhibit the known antifungal proteins. The protein-ligand interaction is studied using AutoDock software. The results from AutoDock can then be analyzed in PyMOL software (Chandrashekar et al., 2019). Another currently used method called genome mining that identifies natural compounds based on their gene cluster sequences has shed a new insight for the future discovery of novel marine compounds (Yang et al., 2020). An outline of the general techniques involved in isolation and characterization of the compound possessing antimycobacterial activity is shown in Figure 1. 


\section{Sea water}

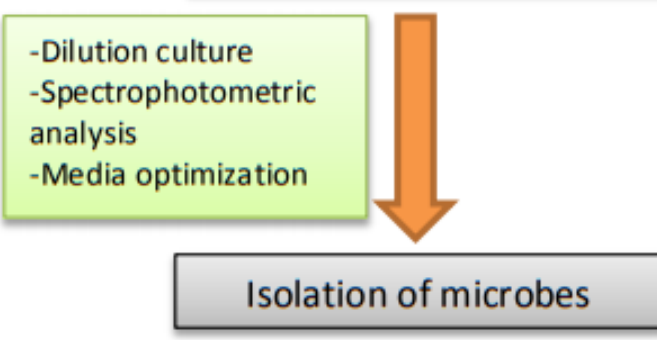

\section{Serial dilution.} Perform spread plate, pour plate, Culture optimization liquid media culture.

\section{Culture identification}

Large scale fermentation

Lyophilization

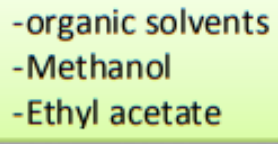

Extraction of compound

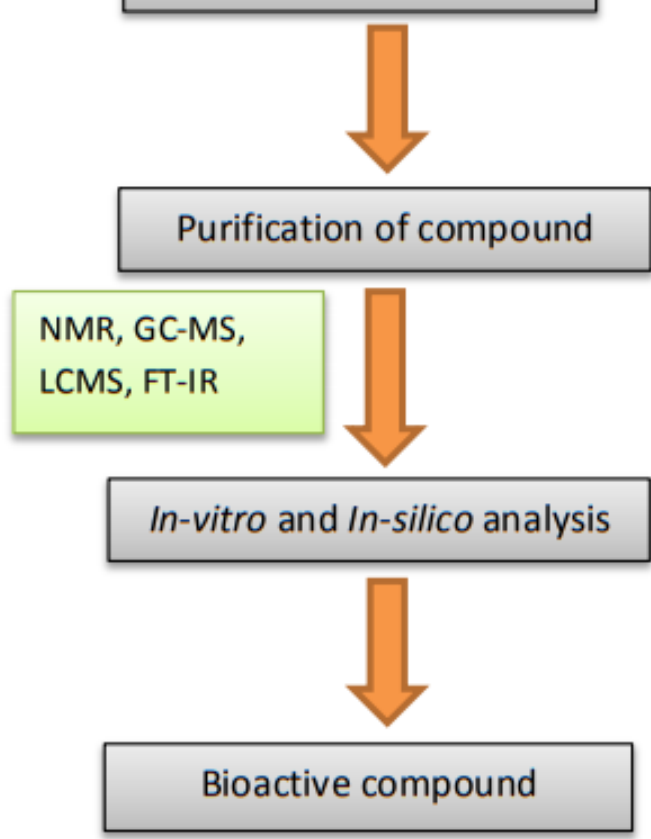

Figure 1. Schematic representation on the main techniques involved in isolation, extraction and characterization of bioactive compound having anti-mycobacterial activity. 


\section{Anti-mycobacterial compounds from marine organisms}

Some of the anti-mycobacterial compounds derived from marine organisms are classified based on their chemical structure include alkaloids, terpenoids, steroids, peptides, polyketides, and pyrones (Figure 2).

\section{Alkaloids}

Structurally diverse alkaloids derived from marine sponges, corals, fungi, algae are known to show anti-mycobacterial activity against $M$. tuberculosis and in some cases also towards resistant strains (Hou et al., 2019). To date, marine sponges of the phylum Porifera are identified as the copious sources of marine bioactive compounds. Manzamines are alkaloids having $\beta$-carboline moiety which was isolated from marine sponges and are known to possess a wide range of biological activity like anti-inflammatory, antimicrobial, cytotoxic, and antiviral activities (De Souza and Marcus, 2006). The presence of hydroxyl group present on the $\beta$-carboline moiety has a major role in the bioactivity of these compounds. Due to their eccentric and distinct layout, they have been attractive leads for pharmacological studies. In particular, compounds such as manzamines A, F, 8hydroxymanzamine A and ircinol A was found to exhibit strong inhibition against $M$. tuberculosis (strain H37RV) with minimum inhibitory concentration (MIC) values of 1.64, 0.69, 2.84 and $4.61 \mu \mathrm{M}$ which further led to the search of more alkaloids (Hou et al., 2019).

In a recent review, Hou and co-workers have isolated halicyclamine alkaloids from marine sponge Haliclona sp. which has been tested against M. tuberculosis. The bactericidal activity of halicyclamines was determined using a colonyforming unit assay (CFU). On the other hand, in the de novo synthesis of guanine nucleotide, the compound was found to be a potent inhibitor of inosine 5'- monophosphate dehydrogenase (IMPDH) converting inosine monophosphate to xanthosine monophosphate. Studies reveal that halicyclamine A hinder the function of DedA protein which has a major role in cell envelope biogenesis in Mycobacterium sp. as well as the responsible gene in conferring resistance (Daletos et al., 2016). The compound also revealed strong bactericidal activity against $M$. smegmatis, $M$. bovis BCG and $M$. tuberculosis (H37Ra) with MIC values ranging from 2.16-10.82 $\mu \mathrm{M}$ respectively (Hou et al., 2019).

Caulerpin, a unique green algal pigment has been isolated from Caulerpa racemosa and $C$. serrulata reported to reveal potent activity against M. tuberculosis $(\mathrm{H} 37 \mathrm{Rv})$ with an $\mathrm{IC}_{50}$ value of 0.24 $\mu \mathrm{M}$. In addition to this, the compound also appears to have a low toxicity profile against human cell lines and possess an ample number of bioactive properties (Chay et al., 2014).

Diketopiperazines which belong to the brevianamide class of alkaloids were extracted from the marine fungus Aspergillus versicolor (Song et al., 2012). They are pervasive and recently came to the light for their vital activities including the disruption of biofilm formation through regulation of LuxR mediated bacterial quorum sensing and cell-cell signaling. Thus, providing alternative ways to control the growth of biofilm by hindering bacterial communication. A few studies indicate that diketopiperazines exhibit strong inhibitory activity against $M$. tuberculosis and are a promising scaffold for next-generation antimycobacterial drugs (De Carvalho and Abraham, 2012).

\section{Terpenoids}

Terpenes, the largest group of secondary metabolites are potential pharmaceutical agents in human disease treatment and precaution. In the past few years, several terpenoids with promising biological activities have been isolated from various marine environments having inhibitory activity against $M$. tuberculosis protein tyrosine phosphatase B (mPTPB). Apart from this, marine terpenoids display novel structural motifs that are derived from biosynthetic pathways unique to marine organisms (Wang et al., 2005). Some of the marine-derived bioactive compounds from terpenoids include sesquiterpenoids isolated from the fungus Emericella variecolor was known to be a potent inhibitor of mycobacterial biofilm formation against $M$. bovis BCG. Another compound named merochlorin A from Streptomyces sp. displayed strong inhibitory activity $\left(\mathrm{IC}_{50}=0.16-2.50\right)$ and low cytotoxicity against $M$. bovis BCG infected macrophage cells (Hou et al., 2019). 


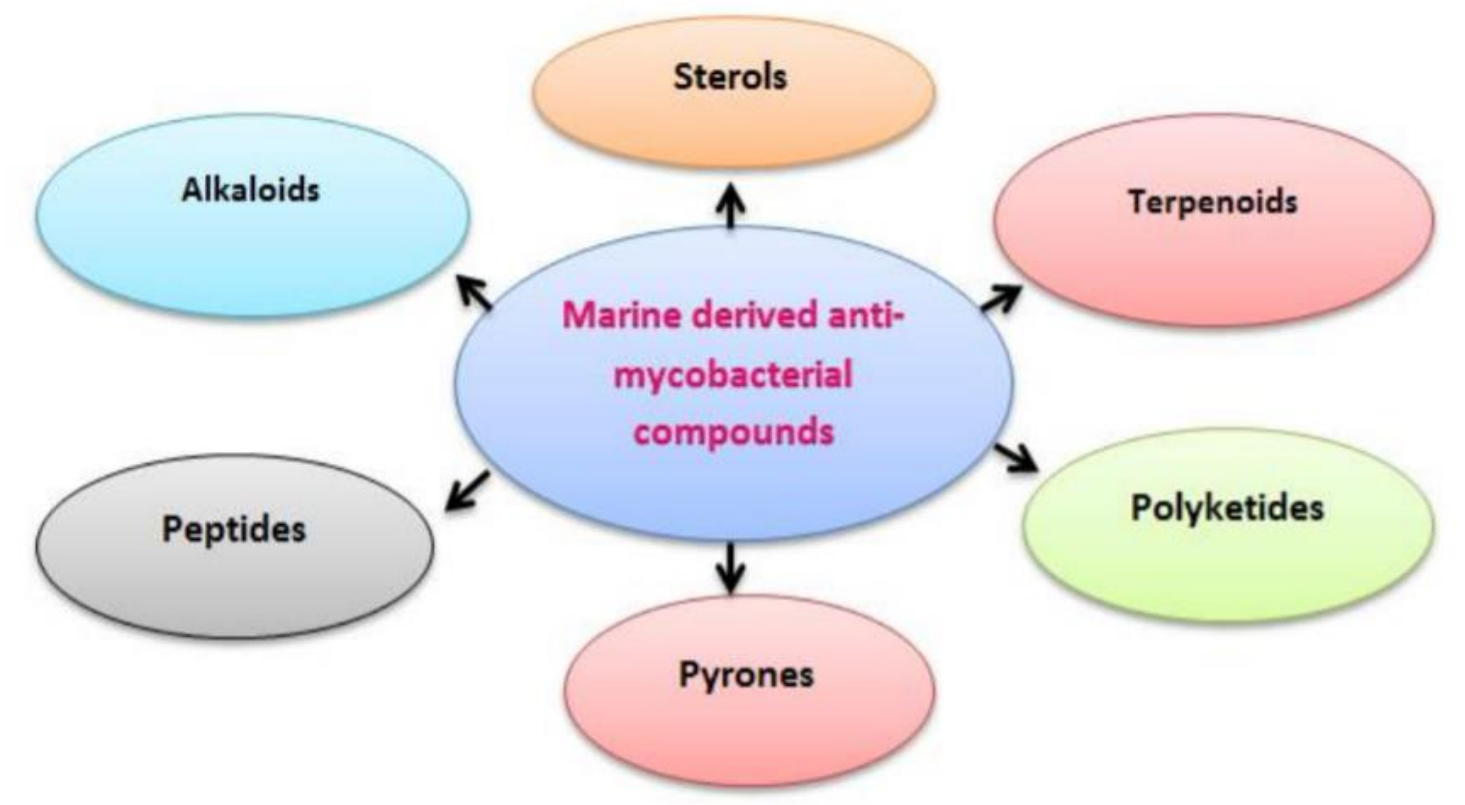

Figure 2. Schematic representation of anti-mycobacterial compounds from marine origin.

\section{Peptides}

Peptides were initially considered unenviable candidates for drug development due to their poor pharmacokinetic properties. However, the advent of new technologies has alleviated the metabolic instability of peptides to potential therapeutics for many deadly diseases. Reports revealed numerous biologically active peptides derived from marine organisms having antimycobacterial activity (Zhang et al., 2019; Rodriguez et al. 2016). Aminolipopeptides and trichoderin derivatives isolated from marine fungus Trichoderma sp. are known to have potent activity against $M$. smegmatis, $M$. bovis BCG, and $M$. tuberculosis strain which was determined from their minimum inhibitory concentration (MIC) values ranging from $0.02-2.0 \mu \mathrm{g} / \mathrm{ml}$ (Garcia et al., 2012). Another marine-derived peptide with antimycobacterial potential is callyaerins isolated from marine sponge Cally spongia aerizusa. The overall structure of callyaerins contains a cyclic peptide segment and a linear peptide side chain connected through a (Z)-2, 3- diaminoacrylamide unit (DAA). Callyaerins contain an unusual proline-rich cyclic peptides which gives them bioactivity to pass through the membranes and specificity to interact with the molecular target. In addition to that, the peptide ring of the compound contains a typical (Z)-2,3- diaminoacrylamide unit (DAA) which gives peptide conformation a peculiar rigidity thereby playing a significant role in the anti- tuberculosis activity (Zhang et al., 2019; Daletos et al., 2016). This bioactive compound was tested for its anti-mycobacterial activity against $M$. tuberculosis strain (MIC $=6.0$ $\mu \mathrm{M})$ and did not show cross-resistance with existing antibiotics (Zhang et al., 2019; Hou et al., 2019). From the few examples listed above, we can say that peptides derived from marine organisms are potential drug candidates due to their high specificity, low immunogenicity, and low toxicity.

\section{Polyketides}

Within the fungal group, polyketides are compounds produced by filamentous fungi. They are structurally complex bioactive compounds containing

hydroxyanthraquinones, flavonoids, and macrolides. These compounds are multifunctional with a wide range of therapeutic uses (Fouillaud et al., 2016). A few literature studies have shown that bostrycin isolated from mangrove endophytic fungus exhibited strong inhibition against multi-drug resistant $M$. tuberculosis strains. Apart from this, bostrycin has multiple targets in mycobacteria. A related study revealed that an effector protein named protein tyrosine phosphatase produced by mycobacterium is needed for the intracellular 
survival of $M$. tuberculosis by hindering host signaling pathways thereby killing mycobacterial survival in macrophages but not affecting the extracellular growth of $M$. tuberculosis (Yuan et al., 2020). Another marine-derived compound anthraquinone isolated from the actinomycete Streptomyces sp. showed inhibitory effects against M. tuberculosis and M. bovis BCG (Supong et al., 2012). Thus polyketide compounds derived from marine fungi provide a new roadmap for research.

\section{Steroids and Pyrones}

Sterols derived from marine organisms are found to have much clinical relevance and are identified as potential therapeutic agents. Mycobacteria are known to possess a sterol biosynthetic pathway which shows homology to that of the yeast. Sterol $14 \alpha$ - demethylase is found to be a potent target in $M$. tuberculosis. Two sterols, abeo-sterols and parguesterols A and B isolated from marine sponge Svenzea reai have promising antimycobacterial activity against mycobacterial strain $\mathrm{H} 3 \mathrm{Rv}$ with MIC values of 7.8 and $11.2 \mu \mathrm{g} / \mathrm{mL}$ while MIC values of abeo-sterols ranges from 3.8$15 \mu \mathrm{g} / \mathrm{mL}$ respectively (Kim et al., 2012; Daletos et al., 2016). It displayed weak cytotoxicity against Vero cell lines, suggesting that it is a good candidate for an anti-mycobacterial drug (Garcia et al., 2012).

Pyrones are a class of heterocyclic compounds isolated from marine organisms. Their unique motifs make them widespread and attractive lead as a pharmaceutical agent. Pyrone based natural compounds target the mycobacterial cell wall and also alter the permeability of the membrane. As a result the bioactive compound can traverse the mycobacterial cell wall interfering with the major processes like replication, RNA synthesis and protein synthesis (Bhat et al., 2017). Few studies reported that penicyrones and verrucosidin which are $\alpha$-pyrone derivatives isolated from a marine fungus Penicillium sp. was known to exhibit effective activity against $M$. tuberculosis (Hou et al., 2019). Similarly, many pyrone derivatives isolated from marine fungus, blue-green algae, and marine sponges are known to possess anti-mycobacterial activity (Bhat et al., 2017; Hou et al., 2019).

\section{Actinobacterial derived compounds}

Actinobacteria, a characteristic of both bacteria and fungi are generally disseminated in terrestrial and marine environments. The marine actinobacteria are less explored compared to terrestrial actinobacteria and possess a wide range of enzyme activities and biochemical reactions. The phylum actinobacteria under actinomycetes are a group of gram-positive, aerobic, and nonmotile bacteria that possess asexual spores (Priyanka et al., 2019; Anandan et al., 2016). They are identified by their high G+C (70-80\%) content and grow as aerial mycelia (Dharmaraj, 2010).

The multifunctional activity profiles of actinobacteria have resulted in the uncovering of novel compounds including some antimycobacterial metabolites. Treatment of the deadly disease caused by Mycobacterium has always been a challenge due to its slow-growing nature and immunological tolerance (Hussain et al., 2020). In this regard, the first drug to be introduced for the treatment of TB was Streptomycin but, the emergence of drug resistance strains led to the development of alternative anti-tubercular agents. Some of the bioactive compounds derived from Actinomycetes sp. include lasalocid, monensin, spirotetronates, lobophorin derivatives, caprazamycin B, sansanmycins, streptcytosine A, etc. (Al-Dhabi et al., 2020). The metal complexes of monensin and lasalocid are active against $M$. tuberculosis and are future leads of new drug design (Jadon et al., 2014). A review published in 2019 conducted structure elucidation analysis of spirotetronates and lobophorin and reported that the nitro-sugar moiety is important for anti-mycobacterial activity (Hou et al., 2019).

\section{CONCLUSION}

Tuberculosis, a global epidemic caused by Mycobacterium tuberculosis poses a major challenge in disease treatment due to the emergence of multidrug resistance and co-infection with HIV. It is estimated that two billion people are infected with the tubercle bacilli worldwide. Every year new cases of tuberculosis appear and millions of people die from this deadly disease. The majority of the cases are reported from poor nations. Among them, India is one of the countries highly affected by TB. As a result, the World Health 
Organization in 1993 proclaimed tuberculosis, a global crisis.

In the current scenario, TB cannot be completely treated because of the lower effectiveness of vaccines, and drug therapy using existing antibiotics. Currently available bactericidal drugs are quite effective in reducing the bacterial burden in sputum for at least four months to have a relapse-free cure. The delayed clearance of TB might be due to the dormant $M$. tuberculosis that arises during infection. Another reason is that the bacterium undergoes metabolic shifts which alter their targets. The presently available drugs pose long term duration and have side effects. Therefore the scientific community is looking forward to novel anti-mycobacterial drugs with new bimolecular targets for the treatment of MDR or XDR Mycobacterium strains with less toxicity and short duration.

Marine organisms are a promising origin of novel metabolites with substantial pharmaceutical and therapeutic potential. Scanty reports are found on in vitro anti-mycobacterial activity from marine origin. However, the bioactive compounds discovered so far have shown potential anti-mycobacterial activity, and some are also effective against multi-drug resistant tuberculosis. Limited studies have identified actinomycetes as an excellent potential source for anti-mycobacterial activity. However, greater study efforts are required to identify more bioactive compounds that can specifically act on mycobacteria.

\section{REFERENCES}

Abrahams, K. A., \& Besra, G. S. 2018. Mycobacterial cell wall biosynthesis: a multifaceted antibiotic target. Parasitology 145(2): 116-133.

Al-Dhabi, N. A., Esmail, G. A., Ghilan, A. K. M., Arasu, M. V., \& Duraipandiyan, V. 2020. Metabolite profiling of Streptomyces sp. Al-Dhabi-100 isolated from the marine environment in Saudi Arabia with anti-bacterial, antitubercular and anti-oxidant potentials. Journal of King Saud University-Science 32(2): 1628-1633.

Anandan, R., Dharumadurai, D., \& Manogaran, G. P. 2016. An introduction to actinobacteria. In Actinobacteria-Basics and Biotechnological Applications. Intechopen.

Arai, M., Niikawa, H., \& Kobayashi, M. 2013. Marine-derived fungal sesterterpenes, ophiobolins, inhibit biofilm formation of Mycobacterium species. Journal of Natural Medicines 67(2): 271-275.
Bhat, Z. S., Rather, M. A., Syed, K. Y., \& Ahmad, Z. 2017. $\alpha-$ pyrones and their hydroxylated analogs as promising scaffolds against Mycobacterium tuberculosis. Future Medicinal Chemistry 9(17): 2053-2067.

Bibi, F., Yasir, M., Al-Sofyani, A., Naseer, M. I., \& Azhar, E. I. 2020. Antimicrobial activity of bacteria from marine sponge Suberea mollis and bioactive metabolites of Vibrio sp. EA348. Saudi Journal of Biological Sciences.

Brinkmann, C. M., Marker, A., \& Kurtböke, D. I. 2017. An overview on marine sponge-symbiotic bacteria as unexhausted sources for natural product discovery. Diversity 9(4): 40.

Chandrashekar, A., Muralidharan, A., Koteshwara, A., Alex, A. T., \& Subrahmanyam, V. M. 2019. Isolation and characterization of an Actinomycete strain producing an antifungal metabolite effective against Candida albicans. Research Journal of Pharmacy and Technology 12(10): 4601-4606.

Chay, C. I. C., Cansino, R. G., Pinzón, C. I. E., Torres-Ochoa, R. O., \& Martínez, R. 2014. Synthesis and anti-tuberculosis activity of the marine natural product caulerpin and its analogues. Marine Drugs 12(4): 1757-1772.

Cook, G. M., Berney, M., Gebhard, S., Heinemann, M., Cox, R. A., Danilchanka, O., \& Niederweis, M. 2009. Physiology of mycobacteria. Advances in Microbial Physiology 55: 81-319.

Daletos, G., Ancheeva, E., Chaidir, C., Kalscheuer, R., \& Proksch, P. 2016. Antimycobacterial metabolites from marine invertebrates. Archiv der Pharmazie 349(10): 763-773.

De Carvalho, M., \& Abraham, W. R. 2012. Antimicrobial and biofilm inhibiting diketopiperazines. Current Medicinal Chemistry 19(21): 3564-3577.

De Souza, M. V. N. 2006. Marine natural products against tuberculosis. The Scientific World Journal 6: 847.

Dharmaraj, S. 2010. Marine Streptomyces as a novel source of bioactive substances. World Journal of Microbiology and Biotechnology 26(12): 2123-2139.

Fouillaud, M., Venkatachalam, M., Girard-Valenciennes, E., Caro, Y., \& Dufossé, L. 2016. Anthraquinones and derivatives from marine-derived fungi: Structural diversity and selected biological activities. Marine Drugs 14(4): 64.

García, A., Bocanegra-García, V., Palma-Nicolás, J. P., \& Rivera, G. 2012. Recent advances in antitubercular natural products. European Journal of Medicinal Chemistry 49: 1-23.

Hou, X. M., Wang, C. Y., Gerwick, W. H., \& Shao, C. L. 2019. Marine natural products as potential anti-tubercular agents. European Journal of Medicinal Chemistry 165: 273-292.

Hussain, A., Hassan, Q. P., \& Shouche, Y. S. 2020. New approaches for antituberculosis leads from Actinobacteria. Drug Discovery Today.

Jadon, R., Singh, V., \& Chaudhary, H. S. 2014. Update on bioactive molecules of actinomycetes. Biosciences Biotechnology Research Asia 11(2): 705-14.

Jankute, M., Cox, J. A., Harrison, J., \& Besra, G. S. 2015. Assembly of the mycobacterial cell wall. Annual Review of Microbiology 69: 405-423.

Kim, S. K., \& Van Ta, Q. 2012. Bioactive sterols from marine resources and their potential benefits for human health. In Advances in Food and Nutrition Research (Vol. 65, pp. 261-268). Academic Press.

Miao, L., Xu, J., Yao, Z., Jiang, Y., Zhou, H., Jiang, W., \& Dong, K. 2017. The anti-quorum sensing activity and bioactive substance of a marine derived Streptomyces. Biotechnology \& Biotechnological Equipment 31(5): 1007-1015.

Nikapitiya, C. 2012. Bioactive secondary metabolites from marine microbes for drug discovery. In Advances in food and nutrition research (Vol. 65, pp. 363-387). Academic Press.

Padhi, A., Naik, S. K., Sengupta, S., Ganguli, G., \& Sonawane, A. 2016. Expression of Mycobacterium tuberculosis NLPC/p60 family protein $\mathrm{Rv} 0024$ induce biofilm formation and 
resistance against cell wall acting anti-tuberculosis drugs in Mycobacterium smegmatis. Microbes and Infection 18(4): 224 236.

Pauli, G. F., Case, R. J., Inui, T., Wang, Y., Cho, S., Fischer, N. H., \& Franzblau, S. G. 2005. New perspectives on natural products in TB drug research. Life Sciences 78(5): 485-494.

Prakash, S., Sasikala, S. L., \& Aldous, V. H. J. 2010. Isolation and identification of MDR-Mycobacterium tuberculosis and screening of partially characterised antimycobacterial compounds from chosen marine micro algae. Asian Pacific Journal of Tropical Medicine 3(8): 655-661.

Priyanka, S., Jayashree, M., Shivani, R., Anwesha, S., \& Rao, K. B. 2019. Characterisation and identification of antibacterial compound from marine actinobacteria: In vitro and in silico analysis. Journal of Infection and Public Health 12(1): 83-89.

Quan, D. H., Nagalingam, G., Luck, I., Proschogo, N., Pillalamarri, V., Addlagatta, A., \& Triccas, J. A. 2019. Bengamides display potent activity against drug-resistant Mycobacterium tuberculosis. Scientific Reports 9(1): 1-10.

Riera-Romo, M., Wilson-Savón, L., \& Hernandez-Balmaseda, I. 2020. Metabolites from marine microorganisms in cancer, immunity, and inflammation: A critical review. Journal of Pharmacy \& Pharmacognosy Research 8(5): 368-391.

Rodriguez, L. M. D. L., Kaur, H., \& Brimble, M. A. 2016. Synthesis and bioactivity of antitubercular peptides and peptidomimetics: an update. Organic \& Biomolecular Chemistry 14(4): 1177-1187.

Sharma, I. M., Petchiappan, A., \& Chatterji, D. 2014. Quorum sensing and biofilm formation in mycobacteria: Role of cdi-GMP and methods to study this second messenger. IUBMB Life 66(12): 823-834.

Siddharth, S. 2019. Isolation and characterization of bioactive compounds with antibacterial, antioxidant and enzyme inhibitory activities from marine-derived rare actinobacteria, Nocardiopsis sp. SCA21. Microbial Pathogenesis 137: 103775.

Smith, I. 2003. Mycobacterium tuberculosis pathogenesis and molecular determinants of virulence. Clinical Microbiology Reviews 16(3): 463-496.

Song, F., Liu, X., Guo, H., Ren, B., Chen, C., Piggott, A. M., \& Liu, X. 2012. Brevianamides with antitubercular potential from a marine-derived isolate of Aspergillus versicolor. Organic Letters 14(18): 4770-4773.

Supong, K., Thawai, C., Suwanborirux, K., Choowong, W., Supothina, S., \& Pittayakhajonwut, P. 2012. Antimalarial and antitubercular $\mathrm{C}$-glycosylated benz $[\alpha]$ anthraquinones from the marine-derived Streptomyces sp. BCC45596. Phytochemistry Letters 5(3): 651-656.

Viegelmann, C., Parker, J., Ooi, T., Clements, C., Abbott, G., Young, L., \& Edrada-Ebel, R. 2014. Isolation and identification of antitrypanosomal and antimycobacterial active steroids from the sponge Haliclona simulans. Marine Drugs 12(5): 2937-2952.

Wang, C., Tang, S., \& Cao, S. 2020. Antimicrobial compounds from marine fungi. Phytochemistry Reviews: 1-33.

Wang, G., Tang, W., \& Bidigare, R. R. 2005. Terpenoids as therapeutic drugs and pharmaceutical agents. In Natural products (pp. 197-227). Humana Press.

Yang, Z., He, J., Wei, X., Ju, J., \& Ma, J. 2020. Exploration and genome mining of natural products from marine Streptomyces. Applied Microbiology and Biotechnology 104(1): 67 76.

Yuan, P., He, L., Chen, D., Sun, Y., Ge, Z., Shen, D., \& Lu, Y. 2020. Proteomic characterization of Mycobacterium tuberculosis reveals potential targets of bostrycin. Journal of Proteomics 212: 103576.
Zhang, S., Kavianinia, I., \& Brimble, M. A. 2019. Naturally occurring antitubercular cyclic peptides. Tetrahedron Letters 60(50): 151339. 